\title{
Aplicação do Teorema do Valor Médio ao Ensino Médio
}

\author{
Edney Freitas Gregorio(1)
}

Nícolas Alcântara de Andrade(i)

\section{Resumo}

Neste trabalho temos por objetivo apresentar o teorema do valor médio como recurso a ser utilizado em provas e demonstrações de algumas afirmações matemáticas. A relevância do trabalho reside na consequência extremamente simples do teorema, ancorada a demonstrações interessantes para alguns teoremas famosos e importantes do ensino médio.

Palavras-chave: Teorema do Valor Médio; Binômio de Newton; Lei de Moivre; Diferença de Potências de Mesmo Expoente; Desigualdade de Bernoulli; Desigualdade de Napier.

\begin{abstract}
In this work we aim to present the mean value theorem as a resource to be used in proofs and demonstrations of some mathematical statements. The relevance of the work lies in the fact that an extremely simple consequence of the theorem, anchored to interesting demonstrations for some famous and important high school theorems.
\end{abstract}

Keywords: Mean Value Theorem; Newton's Binomial; Moivre's Law; Difference of Powers with Same Exponents; Bernoulli Inequality; Napier Inequality

\section{Introdução}

O Teorema do Valor Médio é um dos principais resultados estudados nos cursos de cálculo e análise no ensino superior.

Por sua vez, no ensino médio, dentre outros resultados de destaques, temos:

(i) O Teorema do Binômio de Newton, que permite calcular a expansão binomial e possui aplicações notadamente na teoria da probabilidade.

(ii) A Lei de Moivre, que nos fornece uma maneira prática e rápida de calcular as potências de números complexos.

(iii) A fatoração da diferença de potências de mesmo expoente.

(iv) Desigualdade de Bernoulli, que permite obter uma cota inferior para potências de $(1+\mathrm{x})$.

(v) Desigualdade de Napier, que nos dá informações sobre a regularidade da função lnx. 
Neste artigo vamos apresentar uma demonstração de (i), (ii), (iii), (iv) e (v) a partir do Teorema do Valor Médio, mostrando, assim, que esses resultados, os quais aparentemente não possuem relação, estão ligados entre si.

\section{Teorema do Valor Médio}

O Teorema do Valor Médio, ou Teorema de Lagrange, é um dos principais resultados abordados nos cursos de Cálculo. Apesar de ser um assunto geralmente estudado no ensino superior, ele também costuma ser estudado em turmas do $3^{\circ}$ ano do ensino médio voltadas às olimpíadas e preparatórias para vestibulares como ITA e IME. Como o teorema do valor médio aborda conceitos da geometria analítica, como coeficiente angular e equação da reta, dessa forma ele pode ser aplicado no ensino médio, na resolução de alguns exercícios específicos que envolvem tais conceitos de Geometria Analítica.

Esse teorema foi provado pela primeira vez por Lagrange e, posteriormente, Cauchy apresentou uma forma mais geral dele. Tal resultado, obtido a partir do Teorema de Rolle, foi peça fundamental para a demonstração do Teorema Fundamental do Cálculo.

Podemos enunciá-lo da seguinte forma:

Teorema 1 (Teorema do Valor Médio (TVM)). Seja $\mathrm{f}:[\mathrm{a}, \mathrm{b}] \rightarrow \mathbb{R}$ uma função contínua em $[\mathrm{a}, \mathrm{b}]$ e diferenciável em $(\mathrm{a}, \mathrm{b})$. Então, existe pelo menos um $\mathrm{c} \in(\mathrm{a}, \mathrm{b})$ tal que:

$$
\frac{f(b)-f(a)}{b-a}=f^{\prime}(c)
$$

Tal resultado apresenta a seguinte interpretação geométrica: se uma função y = f(x) é contínua em [a,b] e derivável em $(\mathrm{a}, \mathrm{b})$, então existe pelo menos um ponto c entre os pontos a e b onde a reta tangente à curva é paralela à reta suporte da corda que une os pontos $\mathrm{A}(\mathrm{a}, \mathrm{f}(\mathrm{a}))$ e $\mathrm{B}(\mathrm{b}, \mathrm{f}(\mathrm{b}))$, conforme a figura abaixo.

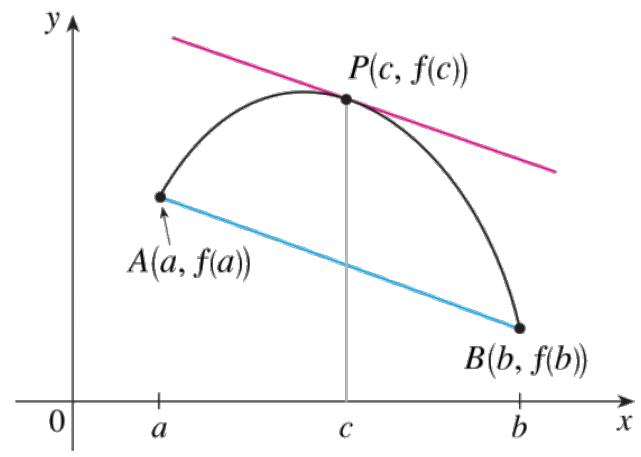

Figura 1: TVM (Teorema do Valor Médio).

Como o teorema do Valor Médio está relacionado à inclinação da reta tangente a uma curva qualquer, vê-se que uma das condições necessárias é que a função que define a curva seja diferenciável 
apenas no intervalo aberto $(\mathrm{a}, \mathrm{b})$, o que equivale a afirmar que a função tem que ser diferenciável apenas nos pontos interiores do intervalo [a,b], ou seja, diferenciável no interior de [a,b]. Vale observar ainda que o ponto c cuja existência é garantida pelo teorema pode não ser único, como vemos na imagem abaixo.

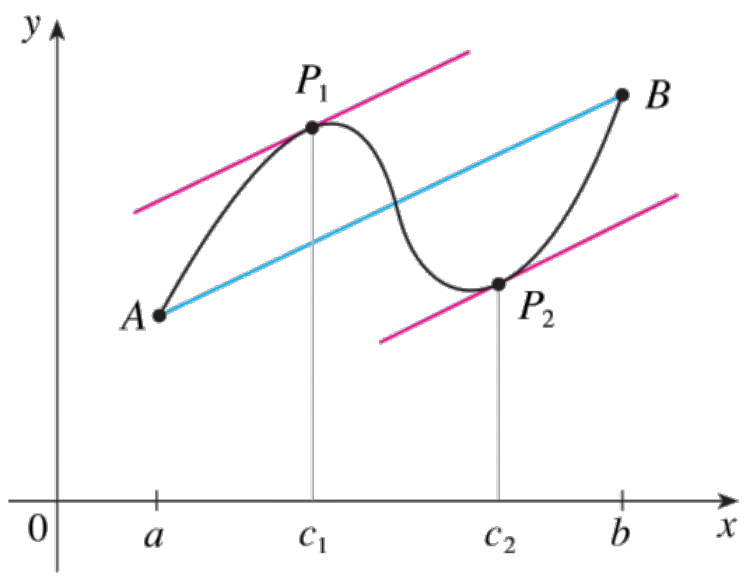

Figura 2: TVM (Teorema do Valor Médio).

O Teorema de Lagrange também pode ser interpretado em termos físicos: supondo que um objeto encontra-se em movimento, e se a sua velocidade média for v durante um determinado percurso, digamos que do ponto a ao ponto b; então, durante esse percurso, ou seja, no intervalo [a, b], existe (pelo menos) um momento em que a sua velocidade instantânea (daqui o conceito de derivada) também é v.

Tal teorema possui várias aplicações importantes, em particular destacamos o seguinte corolário:

Corolário 1. Seja $\mathrm{f}:(\mathrm{a}, \mathrm{b}) \subset \mathbb{R} \rightarrow \mathbb{R}$ uma função diferenciável. Se $\mathrm{f}^{\prime}(\mathrm{x})=0,8 \mathrm{x} \in(\mathrm{a}, \mathrm{b})$, então $\mathrm{f}$ é constante.

No que segue, usaremos o corolário acima para dar demonstrações alternativas para resultados clássicos do ensino médio.

\section{Aplicação ao Ensino Médio}

\subsection{Binômio de Newton}

O desenvolvimento de potências com expoente natural de um binômio é conhecido como Binômio de Newton ou Teorema Binomial. Dessa forma, podem-se desenvolver expressões do tipo $(\mathrm{x}+\mathrm{y})^{\mathrm{n}}$, com $\mathrm{n}$ natural. Tal resultado é amplamente utilizado em conexão com cálculos de probabilidade envolvendo eventos mutuamente exclusivos, onde não importa a ordem de ocorrência de tais eventos.

Teorema 2 (Binômio de Newton). Sejam $\mathrm{x}, \mathrm{y} \in \mathbb{R} e \mathrm{n} \in \mathbb{N}$, então: 


$$
(x+y)^{n}=\sum_{k=0}^{n}\left(\begin{array}{l}
n \\
k
\end{array}\right) x^{k} y^{n-k}
$$

Demonstração. Inicialmente, provaremos que para todo x real vale:

$$
(\mathrm{x}+1)^{\mathrm{n}}=\sum_{\mathrm{k}=0}^{\mathrm{n}}\left(\begin{array}{l}
\mathrm{n} \\
\mathrm{k}
\end{array}\right) \mathrm{x}^{\mathrm{k}}
$$

De fato, defina $f: \mathbb{R} \rightarrow \mathbb{R}$ dada por

$$
f(x)=(x+1)^{n}-\sum_{k=0}^{n}\left(\begin{array}{l}
n \\
k
\end{array}\right) x^{k} .
$$

Observe que f é um polinônio; portanto, $\mathrm{f} \in \mathrm{C}^{\infty}$. Assim, derivando $\mathrm{f}(\mathrm{x})$ obtemos para todo i com $1 \leq \mathrm{i} \leq \mathrm{n}$ :

$$
f^{(i)}(x)=\frac{n !}{(n-i) !}(x+1)^{n-i}-\sum_{k=i}^{n}\left(\begin{array}{l}
n \\
k
\end{array}\right) \frac{k !}{(k-i) !} x^{k-i}
$$

Onde $\mathrm{f}^{(\mathrm{i})}$ é a derivada n-ésima de $\mathrm{f}$ em relação a $\mathrm{x}$.

Daí, fazendo $\mathrm{i}=\mathrm{n}$ na equação acima, obtemos, para todo $\mathrm{x}$ real:

$$
\begin{aligned}
\mathrm{f}^{(\mathrm{n})}(\mathrm{x}) & =\frac{\mathrm{n} !}{(\mathrm{n}-\mathrm{n}) !}(\mathrm{x}+1)^{\mathrm{n}-\mathrm{n}}-\sum_{\mathrm{k}=\mathrm{n}}^{\mathrm{n}}\left(\begin{array}{l}
\mathrm{n} \\
\mathrm{k}
\end{array}\right) \frac{\mathrm{k} !}{(\mathrm{k}-\mathrm{n}) !} \mathrm{x}^{\mathrm{k}-\mathrm{n}} \\
& =\mathrm{n} !-\left(\begin{array}{l}
\mathrm{n} \\
\mathrm{n}
\end{array}\right) \frac{\mathrm{n} !}{0 !} \mathrm{x}^{0} \\
& =0 .
\end{aligned}
$$

Além disso, para todo i, com $1 \leq \mathrm{i}<\mathrm{n}$, temos que:

$$
\begin{aligned}
f^{(i)}(0) & =\frac{n !}{(n-i) !}(0+1)^{n-i}-\sum_{k=i}^{n}\left(\begin{array}{l}
n \\
k
\end{array}\right) \frac{k !}{(k-i) !} 0^{k-i} \\
& =\frac{n !}{(n-i) !}-\left(\begin{array}{c}
n \\
i
\end{array}\right) \frac{i !}{(i-i) !} 0^{0} \\
& =0 .
\end{aligned}
$$

Onde aqui usamos que $\lim _{\mathrm{x} \rightarrow 0^{+}} \mathrm{x}^{\mathrm{x}}=1$

Afirmação 1. Se $\mathrm{f}^{(\mathrm{i})}(\mathrm{x}) \equiv 0$, então, $\mathrm{f}^{(\mathrm{i}-1)}(\mathrm{x}) \equiv 0$. 
De fato, se $\mathrm{f}^{(\mathrm{i})}(\mathrm{x})=0$ para todo $\mathrm{x}$, então, pelo corolário 1 do TVM, $\mathrm{f}^{(\mathrm{i}-1)}$ é constante.Como, porém, $\mathrm{f}^{(\mathrm{i}-1)}(0)=0$, segue que $\mathrm{f}^{(\mathrm{i}-1)}(\mathrm{x})=0$ para todo $\mathrm{x}$ real.

Dessa forma, como $\mathrm{f}^{(\mathrm{n})}(\mathrm{x}) \equiv 0$, segue que $\mathrm{f}^{(\mathrm{i})}(\mathrm{x}) \equiv 0$ para todo i entre 1 e $\mathrm{n}$. Em particular, $\mathrm{f}^{\prime}(\mathrm{x})=0$ para todo $\mathrm{x}$ real, o que nos diz que $\mathrm{f}$ é constante. Como, porém, $\mathrm{f}(0)=0$, segue que $\mathrm{f}(\mathrm{x}) \equiv 0$. Finalmente temos:

$$
0=f(x)=(x+1)^{n}-\sum_{k=0}^{n}\left(\begin{array}{l}
n \\
k
\end{array}\right) x^{k}
$$

E, portanto:

$$
(\mathrm{x}+1)^{\mathrm{n}}=\sum_{\mathrm{k}=0}^{\mathrm{n}}\left(\begin{array}{l}
\mathrm{n} \\
\mathrm{k}
\end{array}\right) \mathrm{x}^{\mathrm{k}} .
$$

Para o caso geral, basta fazer a substituição de $\mathrm{x}$ por $\frac{\mathrm{x}}{\mathrm{y}}$, onde $\mathrm{y} \neq 0$. O que nos dá:

$$
\begin{aligned}
& \left(\frac{\mathrm{x}}{\mathrm{y}}+1\right)^{\mathrm{n}}=\sum_{\mathrm{k}=0}^{\mathrm{n}}\left(\begin{array}{l}
\mathrm{n} \\
\mathrm{k}
\end{array}\right)\left(\frac{\mathrm{x}}{\mathrm{y}}\right)^{\mathrm{k}} \\
& \left(\frac{\mathrm{x}+\mathrm{y}}{\mathrm{y}}\right)^{\mathrm{n}}=\sum_{\mathrm{k}=0}^{\mathrm{n}}\left(\begin{array}{l}
\mathrm{n} \\
\mathrm{k}
\end{array}\right) \mathrm{x}^{\mathrm{k}} \mathrm{y}^{-\mathrm{k}}
\end{aligned}
$$

O que nos diz que

$$
(x+y)^{n}=\sum_{k=0}^{n}\left(\begin{array}{l}
n \\
k
\end{array}\right) x^{k} y^{n-k}
$$

Observação 1 . O caso particular $\mathrm{n}=2$ possui uma interpretação geométrica interessante. 


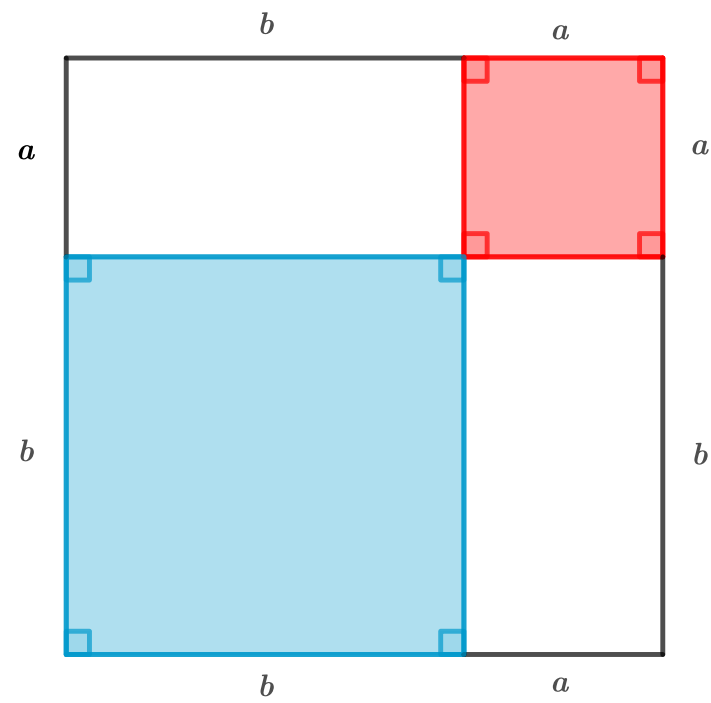

Figura 3: Quadrado da soma de dois termos positivos

Como a figura é um quadrado de lado $\mathrm{a}+\mathrm{b}$, então, sua área é $(\mathrm{a}+\mathrm{b})^{2}$, por outro lado sua área também é igual à soma das áreas do quadrado de lado b, do quadrado de lado a e de dois retângulos congruentes de lados a e b. Totalizando $a^{2}+2 a b+b^{2}$, por fim, sabemos que os dois resultados calculam a mesma área, logo,

$$
(a+b)^{2}=a^{2}+2 a b+b^{2} .
$$

\subsection{Lei de Moivre}

A Primeira Lei de Moivre estabelece as vantagens computacionais da representação polar de números complexos e, além disso, simplifica bastante a obtenção do resultado de uma potência $\mathrm{z}^{\mathrm{n}}=(\mathrm{x}+\mathrm{iy})^{\mathrm{n}}$, já que, para expoentes grandes, resultaria numa decepcionante distribuição de um binômio de Newton.

Teorema 3 (Fórmula de Moivre). Seja $\mathrm{z}=\mathrm{r}(\cos \mathrm{x}+\mathrm{isenx}) \in \mathbb{C}$, com $\mathrm{r}>0, \mathrm{x} \in \mathbb{R}$. Então, para todo $\mathrm{n} \in \mathbb{Z}$, temos que:

$$
\mathrm{z}^{\mathrm{n}}=\mathrm{r}^{\mathrm{n}}(\cos (\mathrm{nx})+\operatorname{isen}(\mathrm{nx}))
$$

Demonstração. Basta provar que

$$
(\cos x+i \operatorname{sen} x)^{n}=(\cos (n x)+i \operatorname{sen}(n x)) .
$$


Defina $f: \mathbb{R} \rightarrow \mathbb{C}$ dada por

$$
f(x)=(\cos (x)+i \operatorname{sen}(x))^{n}-(\cos (n x)+i \operatorname{sen}(n x)) .
$$

Temos que f é diferenciável, pois é combinação de funções diferenciáveis. Assim, calculando a derivada de f, obtemos:

$$
\begin{aligned}
f^{\prime}(x) & =n(\cos x+i \operatorname{sen} x)^{n-1}(-\operatorname{sen} x+i \cos x)-n(-\operatorname{sen}(n x)+i \cos (n x)) \\
& =n(\cos x+i \operatorname{sen} x)^{n-1}(i \operatorname{sen} x+\cos x) i-n i(i \operatorname{sen}(n x)+\cos (n x)) \\
& =n i(\cos x+i \operatorname{sen} x)^{n-1}(\cos x+i \operatorname{sen} x)-n i(\cos (n x)+i \operatorname{sen}(n x)) \\
& =n i(\cos x+i \operatorname{sen} x)^{n}-n i(\cos (n x)+i \operatorname{sen}(n x)) \\
& =n i\left[(\cos x+i \operatorname{sen} x)^{n}-(\cos (n x)+i \operatorname{sen}(n x))\right] \\
& =n i f(x) .
\end{aligned}
$$

Afirmação 2. Afirmamos que $\mathrm{f}(\mathrm{x})=0$ para todo $\mathrm{x} \in \mathbb{R}$.

De fato, pelo visto acima:

$$
\begin{aligned}
\mathrm{f}^{\prime}(\mathrm{x}) & =\operatorname{nif}(\mathrm{x}) \\
\mathrm{e}^{-\operatorname{nix} \mathrm{f}^{\prime}(\mathrm{x})} & =\mathrm{e}^{-\operatorname{nix}} \operatorname{nif}(\mathrm{x}) \\
0 & =\mathrm{e}^{-\operatorname{nix}} \mathrm{f}^{\prime}(\mathrm{x})-\mathrm{e}^{-\operatorname{nix}} \operatorname{nif}(\mathrm{x}) \\
0 & =\left(\mathrm{e}^{-\operatorname{nix}} \mathrm{f}(\mathrm{x})\right)^{\prime} .
\end{aligned}
$$

Para todo $\mathrm{n} \in \mathbb{Z}$ e para todo $\mathrm{x} \in \mathbb{R}$. Assim, pelo corolário 1 do teorema do valor médio aplicado à parte real $\operatorname{Re}(\mathrm{f})$ e imaginária $\operatorname{Im}(\mathrm{f})$ de $\mathrm{f}$, temos que $\operatorname{Re}(\mathrm{f})$ e $\operatorname{Im}(\mathrm{f})$ são constantes e, portanto, a própria $\mathrm{f}$ é constante. Daí, como $\mathrm{f}(0)=0$, segue que $\mathrm{e}^{-\mathrm{nix}} \mathrm{f}(\mathrm{x})=0$ para todo $\mathrm{n} \in \mathbb{Z}$ e para todo $\mathrm{x} \in \mathbb{R}$. Como $\mathrm{e}^{- \text {nix }}$ nunca se anula, segue que $\mathrm{f}(\mathrm{x}) \equiv 0$.

Finalmente,

$$
\begin{aligned}
0 & =f(x) \\
& =(\cos x+i \operatorname{sen} x)^{n}-(\cos (n x)+i \operatorname{sen}(n x)) \\
(\cos x+i \operatorname{sen} x)^{n} & =(\cos (n x)+i \operatorname{sen}(n x))
\end{aligned}
$$

Para todo $n \in \mathbb{Z}$ e para todo $\mathrm{x} \in \mathbb{R}$.

Observação 2. No caso particular para $\mathrm{n}=2,(\cos \mathrm{x}+\mathrm{i} \operatorname{senx})^{2}=\cos (2 \mathrm{x})+\mathrm{i} \operatorname{sen}(2 \mathrm{x})$ podemos obter as fórmulas para o arco duplo do seno e do cosseno. Como as partes reais e complexas de ambos os lados da igualdade devem ser iguais, então, após os cálculos obtemos

$$
\operatorname{sen}(2 x)=2 \operatorname{sen} x \cos x \quad \text { e } \quad \cos (2 x)=\cos ^{2} x-\operatorname{sen}^{2} x
$$




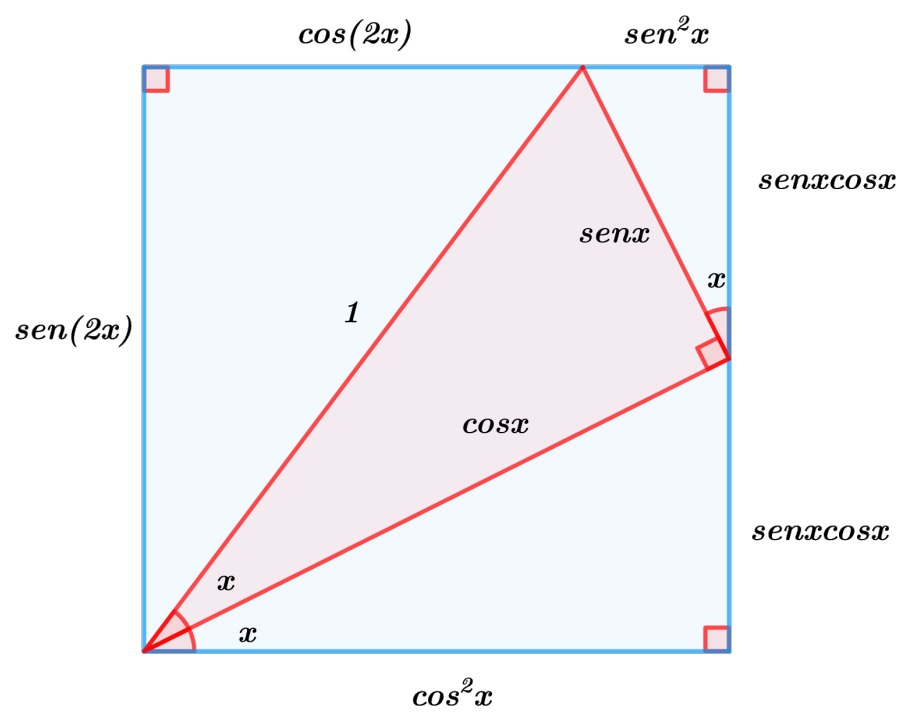

Figura 4: Seno e cosseno do arco duplo para $0<\mathrm{x}<\frac{\pi}{2}$.

\subsection{Diferença de Potências de Mesmo Expoente}

As fatorações constituem uma ferramenta imprescindível para a Matemática, em se tratando de cálculos algébricos. As aplicações envolvem desde o ensino básico até o superior com fatorações importantes para o cálculo diferencial e integral e para a Teoria dos Números.

Teorema 4. Sejam $\mathrm{x}, \mathrm{y} \in \mathbb{R}$ e $\mathrm{n} \in \mathbb{N}$, então:

$$
\mathrm{x}^{\mathrm{n}}-\mathrm{y}^{\mathrm{n}}=(\mathrm{x}-\mathrm{y}) \sum_{\mathrm{k}=0}^{\mathrm{n}-1} \mathrm{x}^{\mathrm{k}} \mathrm{y}^{\mathrm{n}-1-\mathrm{k}}
$$

Demonstração. Provaremos inicialmante que para todo x real vale:

$$
\mathrm{x}^{\mathrm{n}}-1=(\mathrm{x}-1) \sum_{\mathrm{k}=0}^{\mathrm{n}-1} \mathrm{x}^{\mathrm{k}}
$$

Para isso, defina $\mathrm{f}: \mathbb{R} \rightarrow \mathbb{R}$ dada por $\mathrm{f}(\mathrm{x})=\mathrm{x}^{\mathrm{n}}-1-(\mathrm{x}-1) \sum_{\mathrm{k}=0}^{\mathrm{n}-1} \mathrm{x}^{\mathrm{k}}$. Note que $\mathrm{f}$ é diferenciável, pois é um polinômio, daí, derivando $\mathrm{f}(\mathrm{x})$ i vezes $(1 \leq \mathrm{i} \leq \mathrm{n}-1)$, obtemos:

$$
f^{(i)}(x)=\frac{n !}{(n-i) !} x^{n-i}-i \sum_{k=i-1}^{n-1} \frac{k !}{(k-(i-1)) !} x^{k-(i-1)}-(x-1) \sum_{k=i}^{n-1} \frac{k !}{(k-i) !} x^{k-i}
$$

Note que $\mathrm{f}^{\mathrm{n}-1}(\mathrm{x}) \equiv 0$. De fato, para todo $\mathrm{x}$ real: 


$$
\mathrm{f}^{\mathrm{n}-1}(\mathrm{x})=\mathrm{n} ! \mathrm{x}-(\mathrm{n}-1)((\mathrm{n}-2) !+(\mathrm{n}-1) ! \mathrm{x})-(\mathrm{x}-1)(\mathrm{n}-1) !=0 .
$$

Além disso, observe que $\mathrm{f}^{(\mathrm{i})}(0)=0$ para $1 \leq \mathrm{i} \leq \mathrm{n}-1$. De fato:

$$
f^{(i)}(0)=-i(i-1) !-(-1) i !=-i !+i !=0 \text {. }
$$

Finalmente, perceba que se $\mathrm{f}^{(\mathrm{i})}(\mathrm{x})=0$, então, $\mathrm{f}^{(\mathrm{i}-1)}(\mathrm{x}) \equiv 0$. Ora, tal fato é imediato, pois $\mathrm{f}^{(\mathrm{i})}(\mathrm{x})=0$ nos diz que $\mathrm{f}^{(\mathrm{i}-1)}$ é constante pelo corolário 1 do teorema do valor médio. Como, porém, vimos que $\mathrm{f}^{(\mathrm{i}-1)}(0)=0$, segue que $\mathrm{f}^{(\mathrm{i}-1)}(\mathrm{x}) \equiv 0$. Dessa forma, temos que $\mathrm{f}^{\prime}, \mathrm{f}^{\prime \prime}, \ldots, \mathrm{f}^{(\mathrm{n}-1)}$ são todas identicamente nulas; portanto, a própria f é identicamente nula, uma vez que, em particular, $\mathrm{f}^{\prime}(\mathrm{x}) \equiv 0$, o que nos diz que $\mathrm{f}$ é constante. Como, porém, $\mathrm{f}(0)=0$, segue que $\mathrm{f}(\mathrm{x})=0$ para todo $\mathrm{x}$ real. O que nos diz que:

$$
\begin{aligned}
0 & =f(x) \\
0 & =x^{n}-1-(x-1) \sum_{k=0}^{n-1} x^{k} \\
x^{n}-1 & =(x-1) \sum_{k=0}^{n-1} x^{k} .
\end{aligned}
$$

Para o que falta, substitua $\mathrm{x}$ por $\frac{\mathrm{x}}{\mathrm{y}}$, onde $\mathrm{y} \neq 0$, em $(7)$, obtendo:

$$
\begin{aligned}
\left(\frac{x}{y}\right)^{n}-1 & =\left(\frac{x}{y}-1\right) \sum_{k=0}^{n-1}\left(\frac{x}{y}\right)^{k} \\
x^{n}-y^{n} & =(x-y) \sum_{k=0}^{n-1} x^{k} y^{n-1-k} .
\end{aligned}
$$

Observação 3. No caso particular para $\mathrm{n}=2, \mathrm{x}^{2}-\mathrm{y}^{2}=(\mathrm{x}-\mathrm{y}) \sum_{\mathrm{k}=0}^{1} \mathrm{x}^{\mathrm{k}} \mathrm{y}^{1-\mathrm{k}}$ representa a fórmula para a diferença de quadrados. 


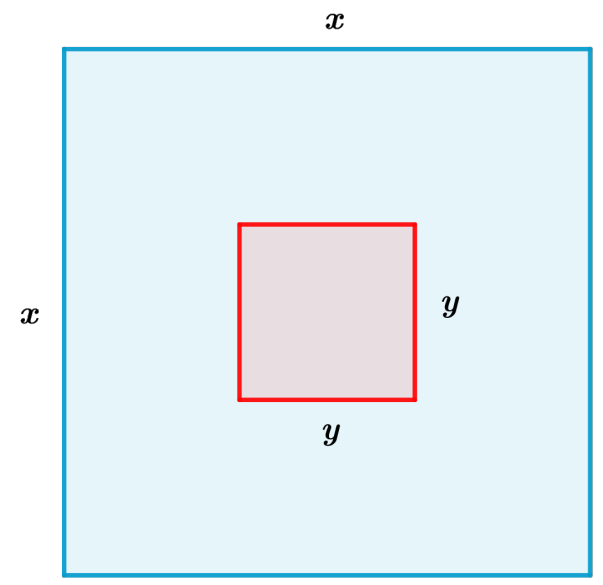

Figura 5: Diferença de dois quadrados de termos positivos.

A Figura 5 é formada por dois quadrados concêntricos de lados paralelos medindo x e y $(\mathrm{x}>\mathrm{y}>0)$. A área da região destacada em azul é a diferença entre a área do quadrado maior e do menor que é igual a $\mathrm{x}^{2}-\mathrm{y}^{2}$; por outro lado, ela também é igual à soma das áreas de quatro trapézios azuis congruentes que são formados ligando os vértices dos lados paralelos dos quadrados. Como cada trapézio tem altura $\frac{x-y}{2}$, base maior x e base menor $y$, segue que a área deles quatro é

$$
4 \frac{\left(\frac{x-y}{2}\right)(x+y)}{2}=(x-y)(x+y) .
$$

Por fim, sabemos que os dois resultados representam a mesma área, logo.

$$
x^{2}-y^{2}=(x-y)(x+y)
$$

\subsection{Desigualdade de Bernoulli}

A desigualdade de Bernoulli é uma relação que permite obter aproximações de potências de $(1+\mathrm{x})$ por um polinômio do primeiro grau. Tal relação foi publicada pela primeira vez por Bernoulli em 1689, e, desde então, tem sido usada frequentemente em várias áreas, como combinatória ou no estudo da convergência de sequecências.

Teorema 5. Se $\mathrm{x}>-1$, então, $(1+\mathrm{x})^{\mathrm{r}} \geq 1+\mathrm{xr}$ para todo $\mathrm{r} \in \mathbb{N}$.

\section{Demonstração.}

(i) Caso $\mathrm{x} \geq 0$ : Considere $\mathrm{f}(\mathrm{t})=(1+\mathrm{t})^{\mathrm{n}}$, para $\mathrm{t} \in[0, \mathrm{x}]$. Assim, f satisfaz as hipóteses do teorema do valor médio, e isso garante a existência de um $c \in(0, x) \operatorname{com} f(x)-f(0)=(x-0) f^{\prime}(c)$. Assim, temos $(1+\mathrm{x})^{\mathrm{r}}-1=\operatorname{xr}(1+\mathrm{c})^{\mathrm{r}-1} \geq \mathrm{xr}$

(ii) Caso $-1<\mathrm{x}<0$ : É feito de forma análoga, considerando $\mathrm{f}(\mathrm{t})=(1+\mathrm{t})^{\mathrm{n}}$, para $\mathrm{t} \in[\mathrm{x}, 0]$. 


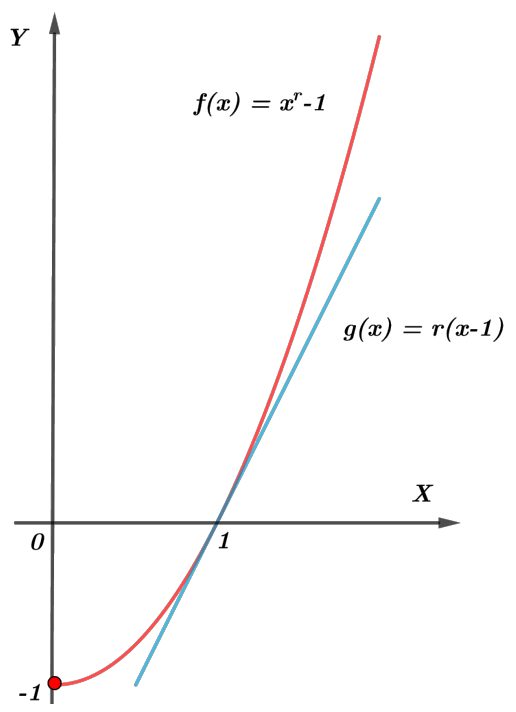

Figura 6: Desigualdade de Bernoulli para $\mathrm{x}>0, \mathrm{x} \neq 1, \mathrm{r}>1 \rightarrow \mathrm{x}^{\mathrm{r}}-1>\mathrm{r}(\mathrm{x}-1)$

\subsection{Desigualdade de Napier}

A desigualdade de Napier constitui uma ferramenta não muito conhecida, porém bastante útil. Ela foi descoberta por John Napier, reconhecido por seus trabalhos evolvendo logaritmos naturais. Dessa forma não é de se espantar que a desigualdade de Napier possa ser usada para dar uma demonstração bem elementar da diferenciabiblidade, e consequente continuidade, da função $\mathrm{f}(\mathrm{x})=\ln \mathrm{x}$.

Teorema 6. Se $\mathrm{b}>\mathrm{a}>0$, então, $\frac{1}{\mathrm{~b}}<\frac{\ln \mathrm{b}-\ln \mathrm{a}}{\mathrm{b}-\mathrm{a}}<\frac{1}{\mathrm{a}}$.

Demonstração. Considere $\mathrm{f}(\mathrm{x})=\ln \mathrm{x}$, para $\mathrm{x} \in[\mathrm{a}, \mathrm{b}]$. Assim, $\mathrm{f}$ satisfaz as hipóteses do teorema do valor médio, e isso garante a existência de $c \in(a, b)$, ou seja, $\frac{1}{b}<\frac{1}{c}<\frac{1}{a}$, com $f(b)-f(a)=f^{\prime}(c)(b-a)$. Assim, temos:

$$
\frac{\ln b-\ln a}{b-a}=\frac{1}{c} \quad \Rightarrow \quad \frac{1}{b}<\frac{\ln b-\ln a}{b-a}<\frac{1}{a}
$$

Para uma interpretação geométrica, considere as retas $\mathrm{L}_{1}, \mathrm{~L}_{2}, \mathrm{~L}_{3}$ onde seus coeficientes angulares são $\mathrm{m}_{\mathrm{L}_{1}}<\mathrm{m}_{\mathrm{L}_{2}}<\mathrm{m}_{\mathrm{L}_{3}}$

$$
\mathrm{m}_{\mathrm{L}_{1}}=\frac{1}{\mathrm{~b}}, \quad \mathrm{~m}_{\mathrm{L}_{2}}=\frac{\ln \mathrm{b}-\ln \mathrm{a}}{\mathrm{b}-\mathrm{a}}, \quad \mathrm{m}_{\mathrm{L}_{3}}=\frac{1}{\mathrm{a}} .
$$

\section{Considerações Finais}




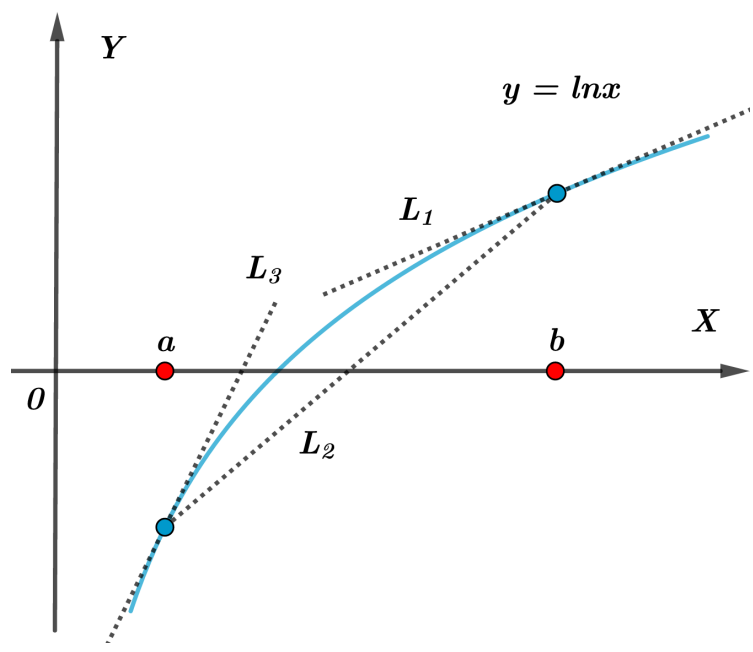

Figura 7: Desigualdade de Napier.

Como vimos, graças ao corolário 1 do Teorema do valor médio, foram apresentadas uma demonstração não indutiva para a primeira lei de Moivre, uma demonstração alternativa para o Binômio de Newton, diferença de potências de mesmo expoente, desigualdade de Bernoulli e desigualdade de Napier. Ressaltamos que um conjunto muito variado de identidades matemáticas pode ser demonstrado por meio desse corolário, em particular, e um exercício simples e interessante para treinar o método abordado aqui seria usá-lo para demonstrar a famosa relação trigonométrica fundamental. Por fim, esperamos que este trabalho possa suscitar outros estudos nesse sentido. 


\section{Referências}

[1] T. Apostol, Calculus, Volume 1, John Wiley \& Sons, Nova Iorque, 1967.

[2] E. L. Lima, Curso de Análise, Volume 1, Impa, Rio de Janeiro, 2000.

[3] R.B. Nelsen, Proofs without words exercises in visual thinking. Volume 1, MAA, 1993.

[4] P.K. Sahoo, Mean Value Theorens and functional equations, World Scientific, 1998

[5] M. Soares, Cálculo em Uma Variável Complexa, Quinta Edição, SBM, Rio de Janeiro, 2016.

Edney Freitas Gregorio Universidade Estadual do Ceará <edney.gregorio@uece.br>

Nícolas Alcântara de Andrade Universidade Estadual do Ceará <nicolas.andrade@uece.br>

Recebido: 11/05/2020

Publicado: 30/07/2020 